\title{
Where Are All the Fallback Disks? Constraints on Propeller Systems
}

\section{Citation}

Ekși, K. Y., L. Hernquist, and R. Narayan. 2005. "Where Are All the Fallback Disks? Constraints on Propeller Systems." The Astrophysical Journal 623 (1): L41-44. https:// doi.org/10.1086/429915.

\section{Permanent link}

http://nrs.harvard.edu/urn-3:HUL.InstRepos:41381817

\section{Terms of Use}

This article was downloaded from Harvard University's DASH repository, and is made available under the terms and conditions applicable to Other Posted Material, as set forth at http:// nrs.harvard.edu/urn-3:HUL.InstRepos:dash.current.terms-of-use\#LAA

\section{Share Your Story}

The Harvard community has made this article openly available.

Please share how this access benefits you. Submit a story.

Accessibility 
DRAFT VERSION OCTOBER 31, 2018

Preprint typeset using $\mathrm{LT}_{\mathrm{E}} \mathrm{X}$ style emulateapj v. 6/22/04

\title{
WHERE ARE ALL THE FALLBACK DISKS? CONSTRAINTS ON PROPELLER SYSTEMS
}

\author{
EKŞI, K.Y., HERNQUIST, L. NARAYAN, R. \\ Harvard-Smithsonian Center for Astrophysics, \\ 60 Garden Street, Cambridge, MA 02138 \\ Draft version October 31, 2018
}

\begin{abstract}
Fallback disks are expected to form around new-born neutron stars following a supernova explosion. In almost all cases, the disk will pass through a propeller stage. If the neutron star is spinning rapidly (initial period $\sim 10 \mathrm{~ms})$ and has an ordinary magnetic moment $\left(\sim 10^{30} \mathrm{G} \mathrm{cm}^{3}\right)$, the rotational power transferred to the disk by the magnetic field of the neutron star will exceed the Eddington limit by many orders of magnitude, and the disk will be rapidly disrupted. Fallback disks can thus survive only around slow-born neutron stars and around black holes, assuming the latter do not torque their surrounding disks as strongly as do neutron stars. This might explain the apparent rarity of fallback disks around young compact objects.

Subject headings: accretion disks_ - pulsars: SN87A—stars: neutron-X-rays: stars
\end{abstract}

\section{INTRODUCTION}

Following a supernova explosion, some of the ejected matter may remain bound to the remnant and fall back (Colgate 1971). A disk can form from this material if its specific angular momentum $l$ exceeds the Keplerian value at the surface of a newly formed neutron star, $l_{\mathrm{K}}=\sqrt{G M R} \simeq 1.4 \times 10^{16} \mathrm{~cm}^{2}$ $\mathrm{s}^{-1}$, where $M$ is the mass of the star and $R$ is its radius. Numerical simulations of the pre-supernova evolution of rotating stars (Heger, Langer \& Woosley 2000) suggest $l \approx 10^{16}-10^{17}$ $\mathrm{cm}^{2} \mathrm{~s}^{-1}$, implying that disk formation is possible (see also Mineshige et al. 1997).

Michel \& Dessler (1981) suggested that fallback disks may be an ingredient of radio pulsars, as an alternative model to the conventional view that pulsars are isolated electromagnetic engines. Lin, Woosley \& Bodenheimer (1991) associated the formation of planets around pulsars with fallback disks. Mever \& Mever-Hofmeister (1989) and Mever-Hofmeister (1992) invoked a fallback disk around the remnant of SN 1987A to explain the deviation of the observed light curve from the theoretical light curve for pure radioactive decay (see also Mineshige, Nomoto \& Shigevama 1993).

More recently, fallback disks (Chatteriee et al. 2000; Alpar 2001; Marsden et al. 2001a; Eksi \& Alpar 2003) have been invoked to explain anomalous X-ray pulsars (AXPs; Woods \& Thompson 2004) via an accretion model rather than the competing magnetar model (Duncan \& Thompson 1992). This model has rekindled interest in the possible implications of fallback disks around radio pulsars. A fallback disk assisting magnetic dipole radiation torque can explain the age discrepancy (Marsden et al. 2001b) and braking indices (Menou et al. 2001) of pulsars, as well as the distribution of radio pulsars on the $P-\dot{P}$ diagram (Alpar et al. 2001). Blackman \& Perna (2004) suggested that the jets observed in the Crab and Vela pulsars are collimated by fallback disks. Oiao et al. (2003) proposed a fallback disk model for periodic timing variations of pulsars. If there are fallback disks assisting the magnetic dipole torque, field estimates based on pure dipole radiation $\left(B \approx 6 \times 10^{19}(P \dot{P})^{1 / 2}\right)$ would be overestimates. Note that the so-called high magnetic field pulsars (Gonzales \& Safi-Harb 2003; Gonzalez et al. 2004; McLaughlin et al. 2003a b) have braking indices less than three, indicating that they are not spinning down purely by emitting magnetic dipole radiation.

A fallback disk (Xu, Wang \& Oiao 2003; Shi \& Xu 2003) has also been invoked for the central compact objects (CCOs; Pavlov. Sanwal \& Teter 2004) in supernova remnants. These objects are likely relatives of AXPs. A typical member of this small group is the central X-ray point source in Cassiopeia A which is just 320 years old and hence likely to possess a fallback disk. A fallback disk model of soft gamma ray repeaters (SGRs; Woods \& Thompson 2004) was invoked by Ertan \& Alpar (2003) who showed that the X-ray enhancement following the August 27 giant flare of SGR 1900+14 can be modeled as the post-burst relaxation of the inner region of a disk which had been pushed back by the flare energy.

While there is ample theoretical motivation for considering fallback disks around young compact objects, the existence of these disks has never been confirmed observationally. There are tight limits on the luminosities of young systems like SN87A (Park 2004) and Cas A (Chakrabarty et al. 2001) where fallback disks are especially likely. For the latter object, Chakrabarty et al. (2001) showed that the optical properties of the source are not similar to those of quiescent low-mass X-ray binaries. Infrared emission is predicted (Perna. Hernquist \& Naravan 2000) by accretion models of AXPs, and possible infrared counterparts have even been identified (see Tam et al. 2004, and references therein) for five of the six known AXPs. The observations are, however, not consistent with a standard thin disk model. Moreover, observations of bursts from AXPs (Gavriil. Kaspi \& Woods 2002) suggest that AXPs are relatives of SGRs for which the magnetar model is favored.

In this letter we address the lack of observational evidence for fallback disks. We argue that fallback disks will be short lived because they would be disrupted by the spin-down power of the neutron star, either in the propeller phase (Shvartsman 1970a; Illarionov \& Sunvaev 1975; Davies \& Pringle 1981; Romanova et al. 2004) or in the radio pulsar phase. In the following section we calculate the spin-down power (Priedhorsky 1986; Shaham \& Tavani 1986; Treves \& Bocci 1987) transferred from the magnetosphere of a neutron star to the disk in the propeller regime and show that for conventional initial periods $P_{0} \sim 10 \mathrm{~ms}$ and magnetic moments ${ }_{\text {mä.harvarđ.edú }} \sim 10^{30} \mathrm{G} \mathrm{cm}^{3}$, as estimated for radio pulsars, the 
luminosity of the disk will be much larger than the Eddington limit for all mass flow rates. In this regime, accretion is highly unstable, and the disk is likely to evolve very quickly until the inner radius of the disk is outside the light cylinder (see $\S 2$ ). Beyond this point, the neutron star becomes a radio pulsar and the star can no longer torque the disk (or vice versa).

In the context of wind-fed, mass-exchange binaries, Shvartsman (1970b) argued that a fast-rotating neutron star would first become an ejector (radio pulsar), in which the disk remains outside the light cylinder. Only after the star slows down can inflowing matter penetrate the light cylinder, allowing the propeller stage to commence. In the case of fallback disks, a fixed amount of mass is available in the disk, rather than a continuous supply of matter as in a massexchange binary. Therefore, once a rapidly rotating neutron star progresses through the initial unstable accretion and propeller stages to an ejector/radio pulsar stage, it is the end of the road for the disk; the system cannot switch back to a propeller or accretion phase. We elaborate upon these points in the following sections.

\section{CONSTRAINTS ON A PROPELLERS}

In order that a fallback disk affect the spin evolution of a neutron star, the inner radius of the disk must be inside the light-cylinder radius $R_{\mathrm{L}}=c / \Omega$ where $\Omega$ is the stellar angular velocity. To within a factor of order unity, the inner radius of the disk, $R_{\mathrm{m}}$, is approximated by the Alfvén radius (Davidson \& Ostriker 1973):

$$
R_{\mathrm{m}} \cong R_{\mathrm{A}} \equiv\left(\frac{\mu^{2}}{\sqrt{2 G M} \dot{M}}\right)^{2 / 7}
$$

where $\dot{M}$ is the mass flow rate and $\mu$ is the stellar magnetic moment. When the disk is inside the light cylinder it suppresses radio pulsar emission. If the inner radius of the disk is also inside the co-rotation radius $R_{\mathrm{c}}=\left(G M / \Omega^{2}\right)^{1 / 3}$, the inflowing matter will accrete and spin the star up.

Initially, a fallback disk is likely to form in the accretion phase. As the $\dot{M}$ in the disk declines (recall that fallback disks are not replenished as are disks in binary systems), the inner radius moves out, and when it goes beyond the co-rotation radius, the system will enter the propeller stage where the inflowing matter, instead of being accreted, is expelled. Here, the neutron star spins down through the interaction of its magnetosphere with the disk. If the torque (angular momentum flux) acting on the star by the disk is $N$, the power transferred to the disk by the neutron star will in turn be $-\Omega N$. This power will add to the gravitational power of the inflowing matter, viz., $G M M \dot{M} / R_{\mathrm{m}}$, enhancing the energy budget of the disk. Some of the energy will be used to drive matter from the system, provided the velocity of the expelled matter, $v_{\text {out }}$, is greater than the escape velocity $v_{\mathrm{esc}}=\sqrt{2 G M / R_{\mathrm{m}}}$. The outflowing matter will carry away kinetic energy at the rate (1/2) $\dot{M} v_{\text {out }}^{2}$. Hence, the net radiative luminosity of the disk in the propeller phase is given by

$$
L_{\mathrm{disk}}= \begin{cases}G M \dot{M} / R_{\mathrm{m}}-\Omega N-\dot{M} v_{\mathrm{out}}^{2} / 2 & \text { if } v_{\mathrm{out}}>v_{\mathrm{esc}}, \\ G M \dot{M} / R_{\mathrm{m}}-\Omega N & \text { if } v_{\mathrm{out}}<v_{\mathrm{esc}} .\end{cases}
$$

The velocity that the expelled matter can attain depends on the coupling of the disk with the magnetosphere; i.e the torque, which can be written as $N=n R_{\mathrm{m}}^{2} \Omega_{\mathrm{K}}\left(R_{\mathrm{m}}\right) \dot{M}$. Here, $n$ is the dimensionless torque and depends only on the dimensionless fastness parameter $\omega_{*}=\Omega / \Omega_{\mathrm{K}}\left(R_{\mathrm{m}}\right)$. Conservation of angular momentum requires that

$$
v_{\text {out }}=R_{\mathrm{m}} \Omega_{\mathrm{K}}\left(R_{\mathrm{m}}\right)(1-n) .
$$

Using this in equation (2) we find that the luminosity of the disk in the propeller regime is

$$
L_{\mathrm{disk}}=\frac{G M \dot{M}}{R_{\mathrm{m}}} \begin{cases}1-\omega_{*} n-(1-n)^{2} / 2 & \text { if } v_{\mathrm{out}}>v_{\mathrm{esc}}, \\ 1-\omega_{*} n & \text { if } v_{\mathrm{out}}<v_{\mathrm{esc}} .\end{cases}
$$

For $v_{\text {out }}<v_{\text {esc }}$ the matter expelled by the magnetosphere can return to the disk at some radius larger than the inner radius. As shown by Spruit \& Taam (1993), the matter will then accumulate near the inner boundary and accrete sporadically.

Many prescriptions have been discussed in the literature for propeller torques. We estimate $n$ by assuming that the magnetosphere and the inflowing matter are "particles" colliding and transferring angular momentum. If the collisions are elastic and the moment of inertia of the star is much larger than that of the inflowing matter one finds $n=2\left(1-\omega_{*}\right)$, whereas completely inelastic collisions would give $n=1-\omega_{*}$. In both cases we assume that the mass of the particle representing the magnetosphere is much greater than that of the particle representing the accreting fluid. Let us thus define an "elasticity parameter" $\beta$ and write

$$
n\left(\omega_{*}\right)=(1+\beta)\left(1-\omega_{*}\right),
$$

where $\beta$ varies between zero and unity and measures how efficiently the kinetic energy of the neutron star is converted into kinetic energy of expelled matter through the interaction of the magnetosphere with the disk. The limiting case of $\beta=0$ corresponds to a "completely inelastic" interaction in which the fraction converted to heat is the maximum, while $\beta=1$ corresponds to an "elastic" interaction in which the rotational energy of the neutron star is converted completely to kinetic energy of expelled matter without heating the disk. This can be seen more clearly when we substitute the torque prescription (5) into equation (4):

$$
L_{\mathrm{disk}}=\frac{G M \dot{M}}{2 R_{\mathrm{m}}} \begin{cases}1+\left(1-\beta^{2}\right)\left(\omega_{*}-1\right)^{2} & \text { if } v_{\mathrm{out}}>v_{\mathrm{esc}}, \\ 2-2(1+\beta)\left(1-\omega_{*}\right) \omega_{*} & \text { if } v_{\mathrm{out}}<v_{\mathrm{esc}} .\end{cases}
$$

For $\beta=1, L_{\text {disk }}=G M \dot{M} / 2 R_{\mathrm{m}}$ which is precisely the luminosity of a Keplerian disk with no torque applied at the inner boundary. Note that Chatteriee et al. (2000) employed $n=2\left(1-\omega_{*}\right)$, referring to the detailed numerical simulations of Daumerie (1996). This corresponds to the limiting case of an "elastic interaction" $(\beta=1)$ in which no heat is produced in the disk by the energy transferred from the neutron star. As any fluid process that strips matter from the disk would be dissipative, the $\beta=1$ limit will never be realized in practice. In the accretion regime, the tangential velocity of the flow will adjust to the velocity of the magnetosphere, which corresponds to $\beta=0$. For the torque to be continuous across the transition from the accretion to propeller regimes, we need to assume $\beta=0$ at least for $\omega_{*} \approx 1$. As the dependence of the expressions in equation (6) on $\beta$ is weak (so long as $\beta$ is not too close to unity), choosing $\beta$ in the range $0-0.5$ produces very little difference in the results. Note that early propeller torques employed in the literature were quite inefficient. In the original work of Illarionov \& Sunvaev (1975) the dimensionless torque was estimated to be $1 / \omega_{*}$. In contrast, the recent detailed simulations of Romanova et al. (2004) suggest even stronger torques than we employ.

From equation (6), we can solve for the minimum period $P_{\min _{1}}$ below which the luminosity of a propeller disk will exceed the Eddington limit, $L_{E}=4 \pi G M c / \kappa_{\mathrm{es}}$, where we take 
$\kappa_{\mathrm{es}}=0.2$ for the electron scattering opacity as appropriate for the high metal content of the fallback matter. Taking $\beta=0$, and defining $\theta=L_{\mathrm{E}} R_{\mathrm{m}} / G M \dot{M}$, we obtain

$$
P_{\min _{1}}=\frac{2 \pi R_{\mathrm{m}}^{3 / 2}}{\sqrt{G M}} \begin{cases}(\sqrt{2 \theta-1}+1)^{-1} & \text { if } v_{\mathrm{out}}>v_{\mathrm{esc}}, \\ (1 / 2+\sqrt{\theta-3 / 4})^{-1} & \text { if } v_{\mathrm{out}}<v_{\mathrm{esc}} .\end{cases}
$$

Another limiting period $P_{\min _{2}}$ is obtained by the condition that the inner radius of the disk be inside the light cylinder. This gives

$$
P_{\min _{2}}=\frac{2 \pi}{c} R_{\mathrm{m}} .
$$

Finally, if the disk is in the accretion phase, then the maximum $\dot{M}$ is given by the Eddington limit: $G M \dot{M}_{\max } / R=L_{\mathrm{Edd}}$. Figure 1 shows all these limits in the $\dot{M}-P$ plane. We see that only a limited area of the plane is consistent with the various constraints we have discussed. Note that, for a given stellar magnetic moment, there is a global minimum period $P_{\min }$ below which no stable disk is permitted for any $\dot{M}$. A neutron star that is born with a shorter period cannot possibly have a stable fallback disk. For $\mu=10^{30} \mathrm{Gcm}^{3}$, the global minimum period is $P_{\min }=35.2 \mathrm{~ms}$. For a general $\mu$ and $R_{\mathrm{L}} \gg R_{\mathrm{c}}$, the minimum period can be approximated as

$$
P_{\min } \approx 0.036 \mu_{30}^{4 / 7} \mathrm{~s}
$$

where $\mu_{30}=\mu / 10^{30}$.

The propeller regime with super-critical mass flow rates has been studied by Mineshige, Rees \& Fabian (1991) who argued that the inner radius of the disk will adjust such that the luminosity does not exceed the Eddington limit. Our work shows that for a neutron star with standard parameters, $\mu=10^{30} \mathrm{G} \mathrm{cm}^{3}, P=10 \mathrm{~ms}$, there is no way for the disk to penetrate the light cylinder and yet have its luminosity be less than the Eddington limit. In such a system, we argue that the accretion and propeller phases will progress very rapidly, on a much shorter time scale than the viscous time of the disk, as the accretion and spin-down power cause the inner regions of the disk to evaporate. Once the disk moves outside the light cylinder, conventional wisdom (Shvartsman 1970b) suggests that the disk would be disrupted by the radiative pressure of the neutron star which now acts like a radio pulsar. Recently, Eksi \& Alpar (2005) argued that the transition from the inner zone dipole field to the radiative zone field can be broad, especially if the inclination angle between the rotation and magnetic field axes is small, which would imply that the disk may be able to find a stable inner boundary outside the light cylinder. If this is the case, the pulsar would evaporate the disk slowly by the interaction of the pulsar wind with the disk. Whether the pulsar will be slowed down to periods where the inner radius of the disk can re-penetrate the light cylinder depends on the complex physics of this interaction.

\section{DISCUSSION}

We have shown in this paper that fallback disks around newborn neutron stars with conventional periods and magnetic moments would be disrupted by a combination of the accretion power and the rotational power of the neutron star being transferred to the disk. Therefore, even though fallback disks may well form, they would not be able to survive for standard initial neutron star parameters.

Our results are general, in the sense that for all propeller systems with parameters outside the shaded region in Figure 1 the disk is either outside the light cylinder or will be pushed there rapidly. Thus, not all combinations of the magnetic moment and period are allowed. In particular, for a given magnetic moment, there is a minimum allowed neutron star period $P_{\min }$ given by equation (9). If a neutron star is born with a shorter period that this, only a very short-lived fallback disk is possible. This might explain why there has been no direct observational evidence for fallback disks around young neutron stars even though one expects a fair amount of fallback material with angular momentum to accumulate during and soon after the supernova explosion.

The recent interest in the fallback disk model is because of the potential application of this model to AXPs. An advantage of this model is that the neutron stars in AXPs are drawn from the same population as radio pulsars, except for one new parameter describing the initial mass of the disk (Chatteriee \& Hernquist 2000). However, our present results indicate that, if fallback disks are to be present around AXPs, these neutron stars must have been born with long periods and/or small magnetic dipole fields that are not typical of radio pulsars. It is interesting that recent studies (Vranesevic et al. 2004) based on the Parkes multibeam survey imply that $40 \%$ of pulsars are "injected" with initial periods in the range $0.1-0.5 \mathrm{~s}$ (as first suggested by Vivekanand \& Naravan 1981, using a smaller sample of pulsars). For the fallback model to be relevant, AXPs must be drawn from this sub-population of slow injected pulsars.

For SN87A, Ögelman \& Alpar (2004) assumed that the compact star was born spinning rapidly and that its present luminosity is due to pure magnetic dipole spin-down. They concluded that the observed upper limit on the luminosity of the star requires the magnetic field of the putative pulsar to be either very small (almost in the millisecond pulsar range) or very large (in the magnetar range). In the latter case, the pulsar has spun down rapidly after the supernova explosion, and so the present luminosity is low. The magnetar solution branch of Ogelman \& Alpar (2004) falls in the region of Figure 1 in which a fallback disk is not allowed. Therefore, their solution is viable; even if the system had fallback material it would have been ejected early on. However, the second solution branch of Ogelman \& Alpar (2004) corresponds to a small magnetic dipole moment $\left(\mu_{30} \sim 0.01\right)$ for the neutron star, so that a fallback disk is very likely to survive. The luminosity from the disk in the propeller phase would then be much larger than the magnetic dipole luminosity assumed by Ögelman \& Alpar (2004). Therefore, the upper limit on the magnetic field must be much lower in order to satisfy the observational constraint on the luminosity.

An alternative explanation for the lack of fallback disks is given by Fryer, Colgate \& Pinto (1999), who argued that accretion flows with heavy elements would be dynamically unstable when the recombination of heavy elements commences, giving rise to a line-driven wind that eventually expels all the initially bound material.

Finally, we note that supernova explosions may sometimes lead to black holes. The formation of fallback disks around black holes was investigated by Mineshige et al. (1997), and a fallback disk model for ultraluminous X-ray sources (ULXs; Fabbiano \& White 2004) was suggested by Li (2003). Rotating black holes may transfer energy to their surrounding disks (e.g., Li 2002), just like neutron stars, in which case such disks would again tend to reach super-Eddington luminosities and be disrupted. However, if the energy transfer is significantly less than in neutron stars, the disk might find it easier 
to survive. It would be interesting to search for fallback disks around young black hole systems. Li (2003) mentions two ULXs associated with supernova remnants that could be such accreting black holes.
We thank M.Ali Alpar and Ünal Ertan for useful discussions. This work was supported in part by NASA grant NNG04GL38G.

\section{REFERENCES}

Alpar, M. A. 2001, ApJ, 554, 1245.

Alpar, M. A., Ankay, A., \& Yazgan, E. 2001, ApJ, 557, L61

Blackman, E. G., \& Perna, R., 2004, ApJ, 601, L71

Chakrabarty, D., Pivovaroff, M. J., Hernquist, L. E., Heyl, J. S., \& Narayan, R., 2001, ApJ, 548, 800

Chatterjee, P. \& Hernquist, L., ApJ, 543, 368

Chatterjee, P., Hernquist, L., \& Narayan, R. 2000, ApJ, 534, 373

Colgate, S. A., 1971, ApJ, 163, 221.

Daumerie, P., PhD thesis, 1996, Illinois Univ.

Davidson, K., \& Ostriker, J. P., 1973, ApJ, 179, 585

Davies, R. E., \& Pringle, J. E., 1981, MNRAS, 196, 209.

Duncan, R.C., \& Thompson, C., 1992, ApJ, 392, L9

Ekşi, K. Y., \& Alpar, M. A., 2003, ApJ, 599, 450.

Ekşi, K. Y., \& Alpar, M. A., 2005, ApJ, in press, astro-ph/0409408

Ertan, Ü., \& Alpar, M. A., 2003, ApJ, 593, L93

Fryer C.L., Colgate S. A., \& Pinto P. A., 1999, ApJ511, 885

Fabbiano, G. \& White, N.E., 2004, in "Compact Stellar X-ray Sources", eds. W.H.G. Lewin and M. van der Klis, Cambridge Univ. Press.

Gavriil, F. P., Kaspi, V. M., \& Woods, P. M., 2002, Nature, 419, 142

Gonzalez, M., \& Safi-Harb, S., 2003, ApJ, 591, L143

Gonzalez, M. E., Kaspi, V. M., Lyne, A. G., \& Pivovaroff, M. J., 2004, ApJ, 610, L37

Heger, A., Langer, N., \& Woosley, S. E. 2000, ApJ, 528, 368

Illarionov, A. F., \& Sunyaev, R.A., 1975, A\&A, 39, 185.

Li, X.-D., 2003, ApJ, 596, L199.

Li, X.-L, 2002, ApJ, 567, 463

Lin, D. N. C., Woosley, S. E., \& Bodenheimer, P. H., 1991, Nature, 353, 827

McLaughlin, M. A., et al., 2003a, in Young Neutron Stars and Their Environments, IAU Symposium, Vol. 218, 2004, F. Camilo and B. M. Gaensler, eds. astro-ph/0310455

McLaughlin, M. A., et al., 2003b, ApJ, L135

Marsden, D., Lingenfelter, R. E., Rothschild R. E., \& Higdon, J. C., 2001a, ApJ, 550, 397.

Marsden, D., Lingenfelter, R. E., \& Rothschild R. E., 2001b, ApJ, 547, L45. Menou, K., Perna R., \& Hernquist, L. 2001, ApJ, 554, L63.
Meyer-Hofmeister, E., 1992, A\&A, 253, 459

Meyer, F., \& Meyer-Hofmeister, E., 1989, in Theory of Accretion Disks, NATO ARW, eds. F. Meyer et al., Kluwer Academic Publishers, p. 307.

Michel, F. C., \& Dessler, A. J., 1981, ApJ, 251, 654

Mineshige, S., Nomoto, K., \& Shigeyama, T., 1993, A\&A, 267, 95

Mineshige, S., Nomura, H., Hirose, M., Nomoto, K., \& Suzuki, T., 1997, ApJ, 489, 227.

Mineshige, S., Rees, M. J., \& Fabian, A. C., 1991, MNRAS, 251, 555

Ögelman, H., \& Alpar, M. A., 2004, ApJ, 603, L33

Park, S., 2004, Adv. Sp. Res., 33, 386

Pavlov, G. G., Sanwal, D., \& Teter, M. A., 2003, in Young Neutron Stars and Their Environments, IAU Symposium, Vol. 218, 2004, F. Camilo and B. M. Gaensler, eds. astro-ph/0311526

Perna, R., Hernquist, L., \& Narayan, R., 2000, ApJ, 541, 344

Priedhorsky, W., 1986, ApJ, 306, L97

Qiao, G. J., Xue, Y. Q., Xu, R. X., Wang, H. G., \& Xiao, B. W., 2003, A\&A, 407, L25

Romanova, M. M., Ustyugova, G. V., Koldoba, A. V., \& Lovelace, R. V. E. 2004 ApJ616, L151

Shaham, J., \& Tavani, M., 1986, IAU Symposium 125, The Origin and Evolution of Neutron Stars, ed. D. J. Helfand and J. H. Huang (Dordrecht: Reidel), p. 199

Shi, Y., \& Xu, R.X., 2003, ApJ, 596, L75

Shvartsman, V. F., 1970, Radiofizika, 13, 1852

Shvartsman, V. F., 1970, Astron. Zh., 47, 660

Spruit, H. C, \& Taam, R. E., 1993, ApJ, 402, 593

Tam, C. R., Kaspi, V. M., van Kerkwijk, M. H., \& Durant, M., ApJ, 617, L53

Treves, A. \& Bocci, F., 1987, MNRAS, 225, Short Communication, 39

Vivekanand, M., \& Narayan, R., 1981, J. Astrophys. Astr. 2, 315

Vranesevic, N. et al. 2004, ApJ, 617, L139

Woods, \& P. M., Thompson, C., 2004, in "Compact Stellar X-ray Sources", eds. W.H.G. Lewin and M. van der Klis, Cambridge Univ. Press.

Xu, R. X, Wang, H. G., Qiao, G. J., 2003, Chin. Phys. Lett., 2, 314 

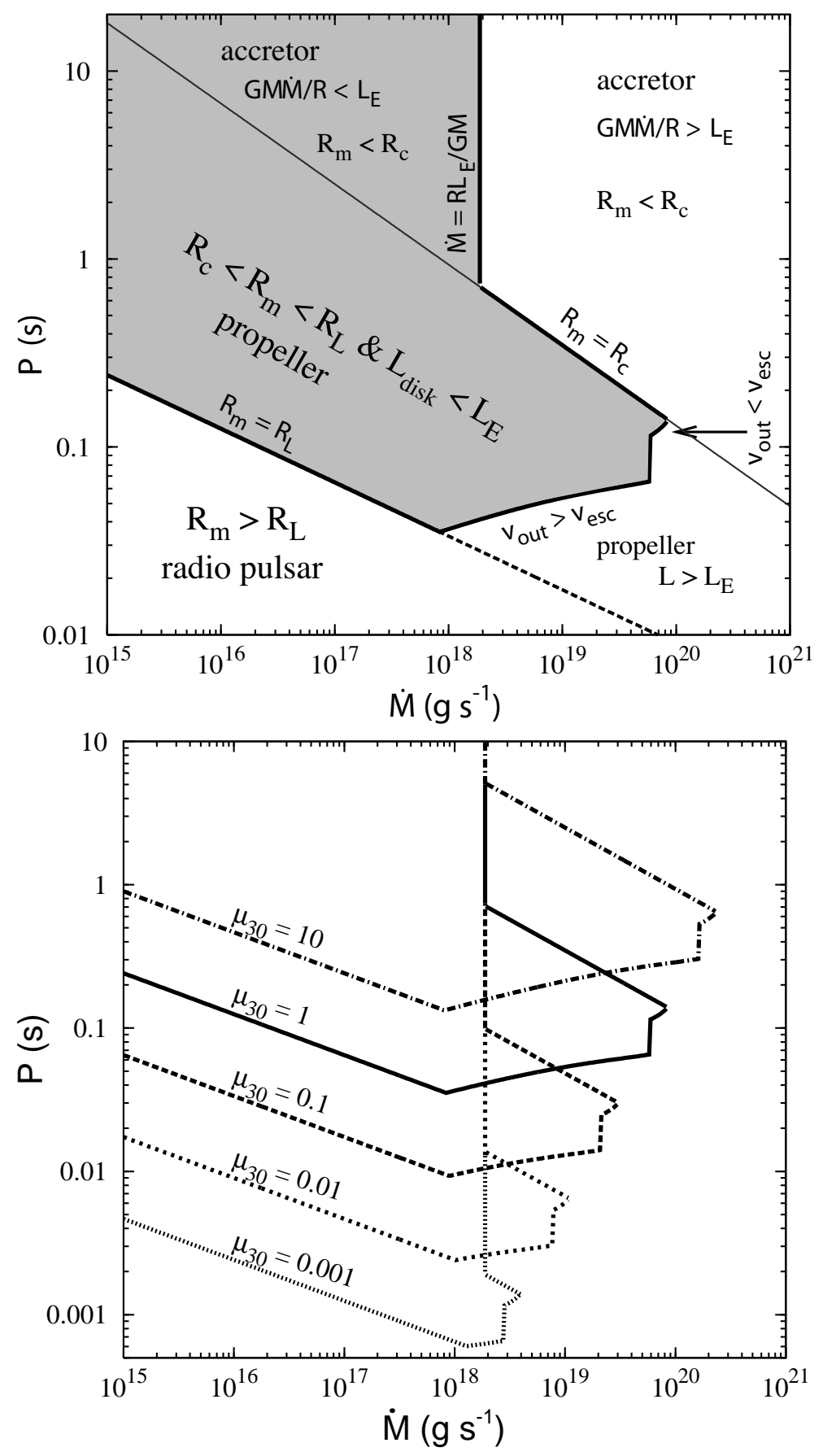

FIG. 1. - First Panel: Allowed regions (shaded) and disallowed regions (not shaded) in the (spin period $P$ )-(mass accretion rate $\dot{M}$ ) plane for an accretion disk around a spinning magnetized star with a magnetic moment $\mu=10^{30} \mathrm{G} \mathrm{cm}^{3}$. The boundary between the two regions is demarcated by various lines corresponding to (i) $R_{m}=R_{L}$ which separates the radio pulsar and propeller phases, (ii) $R_{m}=R_{c}$ which separates the propeller and accretion phases, and (iii) $L=L_{E}$ which separates the sub-Eddington and super-Eddington accretion phases. Note that there is a minimum allowed period $P_{\min }$ which is independent of $\dot{M}$, and a maximum accretion rate which is independent of $P$. Second Panel: Shows the boundaries between the allowed and disallowed regions in the $P-\dot{M}$ plane for several choices of $\mu$. The minimum allowed period $P_{\min }$ scales with $\mu$ as in equation 9 . 